Der Name "Niobe" ist durch die Mehrzahl der Herren Astronomen, welche am $20^{\text {sten }}$ und $21^{\text {sten }}$ August in Dresden versammelt waren, gewählt worden und kommt vor in Homer's Ilias XXIV. 602, 606; Apollodor III. 5, 6; Hygin. fab. 9 und 10; Ovid Met. VI. 148, 156, 165, 273 etc.

Bilk bei Düsseldorf 1861 Aug. 30 .

R. Luther.

Einiges über Pseudo-Daphne (56), von Herrn Dr. R. Euther.

Herr Goldschmidt, der sich mit mir in die Wieder-Aufsuchung seines Planeten Pseudo-Daphne 50 so getheilt hatte, dass er vorzugsweise zwischen Hyp. I. u. II. der in Mis10 gegebenen Ephemeriden suchte, während ich die grösseren Rectascensionen von $21^{\mathrm{h}} 12^{\mathrm{m}}$ bis $22^{\mathrm{h}} 28^{\mathrm{m}}$ durchmustert habe, hat am 27 ten August den wohlverdienten Lohn für seine grosse Ausdauer darin gefunden, dass er den Planeten mit der sternreichen akademischen Charte $20^{\text {h }}$ des Herrn Dr. Hencke gleichsam aufs Neue entdeckt hat. Die tägliche Bewegung zeigt eine befriedigende Ubereinstimmung zwischen Beobachtung und Rechnung, und der Ort des Planeten ist nach der Beobachtung nur $6^{\prime}$ nördlich von der Curve, die aus meinen Elementen folgen würde.

Folgende nur ganz lüchtig entworfene

$$
\text { Elemente } 111 \text {. (36) }
$$

Epoche 1857 Sept. $130^{\text {h }}$ Berlin

$$
\begin{aligned}
& \boldsymbol{M}=36^{\circ} 9^{\prime} 5 \\
& \boldsymbol{\pi}=29440,6 \\
& \delta=19439,5 \\
& \boldsymbol{i}=83,5 \\
& \phi=137,6 \\
& \boldsymbol{\mu}=846^{\prime \prime}, 72
\end{aligned}
$$

hatten bloss den Zweek, die Beobachiungen von 1857 und 1861 vorläufig zu verbinden, und zeigen zugleich die Verschiedenheit des Planeten (66 von Daphue.

Folgende ebenfalls nur flüchtig berechnete Ephemeride wird die Rectascensionen wahrscheinlich etwas zu klein geben:

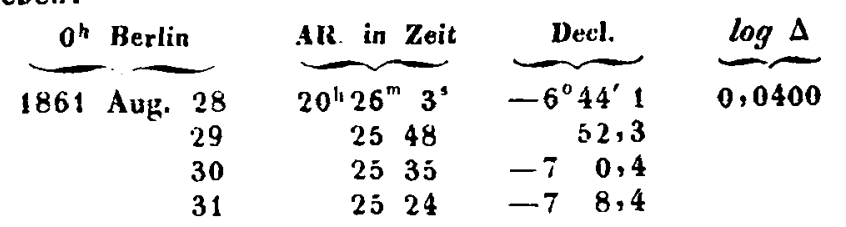

Bilk bei Düsseldorf 1861 Sept. 4.

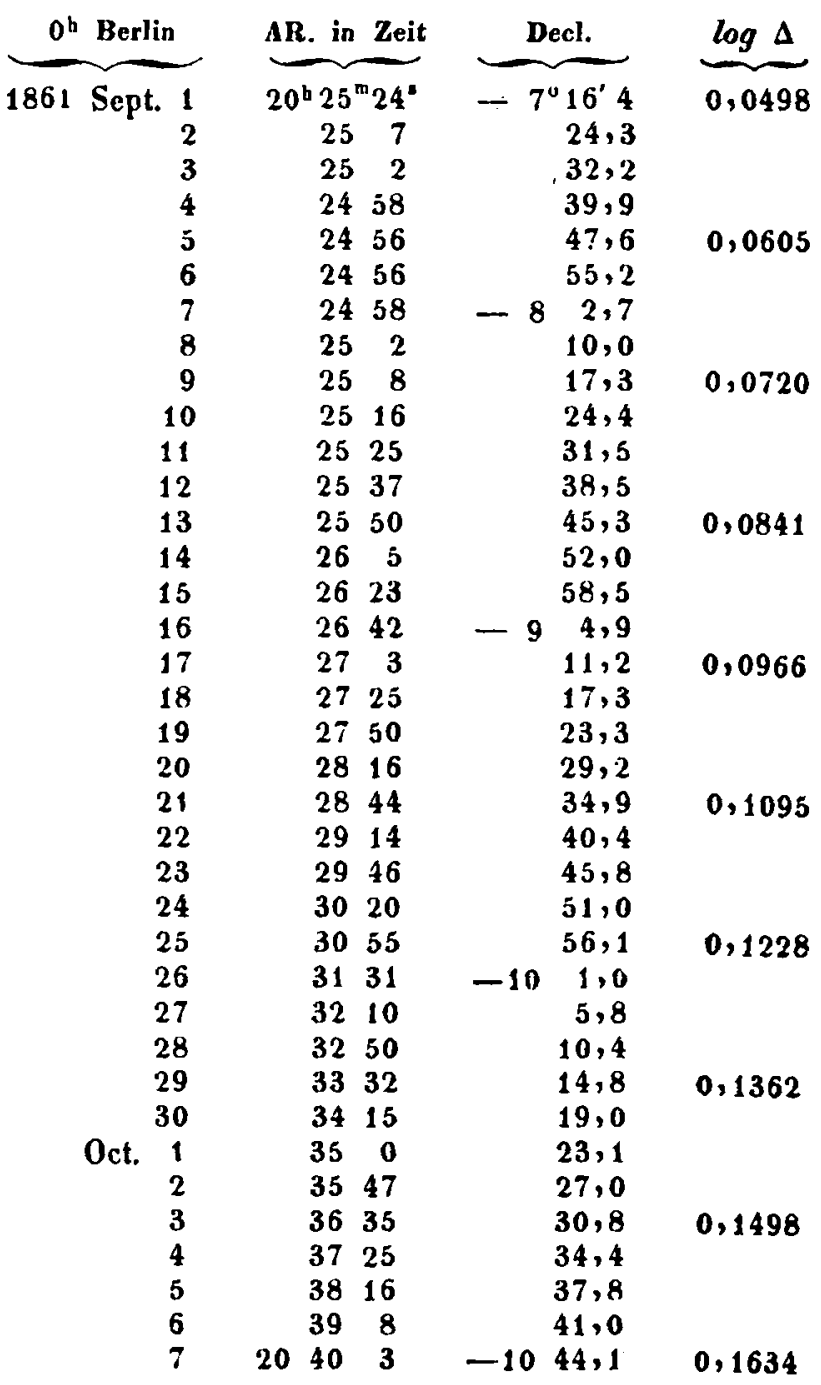

R. Luther.

Elemente des Cometen II. 1861, von Herrn Awwers.

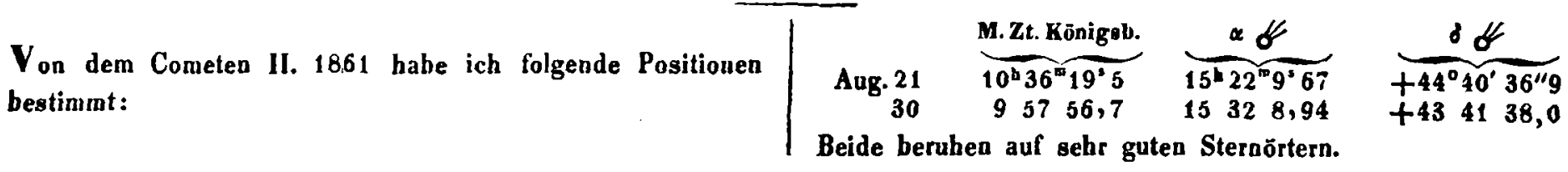


An die erste dieser Beobachtungen. die erste von Liais und das Mittel aus der Pariser und Bilker rom 18ten Juli versuchte ich vergeblich eine Parabel anzuschliessen; der mittlere Ort liess sich nur bis auf $1^{\prime}$ darstellen. Durch Variation von $M$ und $\frac{1}{a}$ ergab sich vielmehr folgende Ellipse:

$$
\begin{aligned}
& T:=\text { Juni 11,55058 Greenwich } \\
& \left.\begin{array}{l}
\pi=249^{\circ} 7^{\prime} 15^{\prime \prime} 4 \\
\Omega=278 \quad 57 \quad 30,0
\end{array}\right\} \text { Mittl. Äquin. } 1861 \\
& i=852918,5 \\
& \log q=9,915033 \\
& \log e=9,995242 \quad \text { Bew. direct. } \\
& \text { Umlaufszeit } 656 \text { Jahre. }
\end{aligned}
$$

Nach Vollendung dieser Rechnung wurden niir die Beobachtungen von Moesta bekannt. Wie diese in dem Pariser Bulletin angageben sind, enthalten sie mehrere Fehler, deren Verbesserung indess zweifellos ist. Juni 10 ist nämlich der Comet mit dem Sterne A.Z. 322, No 108, verglichen, dessen Declination für 1861,0 nach Argelander - $27^{\circ} 53^{\prime} 28^{\prime \prime} 6$ ist, während Lal. 7574 für denselben $-27^{\circ} 52^{\prime} 34^{\prime \prime} 8$ giebt; die Vergleichung der Beobachtung vom 10 ten Juni mit der von $12^{\text {ten }}$ und den Liais'schen zeigt nun, dass die von Moesta benutzte Decl, der Zonen um $+1^{\prime}$ zu corrigiren ist. Ferner

Köuigsberg 1861 Sept. 2. ist Juni $12 \Delta \delta+$ statt - zu nehmen. Nach Anbringung dieser Verbesserungen habe ich die Beobachtungen Santiago Juni 10 und 12, und Rio Janeiro Juni 11 und 13 in den Normalort vereinigt

$$
\text { Juni } 12,0 \text { Gr. } \alpha \not \&=61^{\circ} 14^{\prime} 7^{a} 6 \quad \delta \& \&=-27^{\circ} 18^{\prime} 59^{\prime \prime} 8
$$
mit Ausschluss jedoch der stärker abweichenden und bei einer $\Delta \alpha$ von $7^{\mathrm{m}}$ wobl weniger sicheren AR. von Juni 10 .

Die Beolachtungen Bilk Juli 22 und London Juli 23 gebeu den zweiten Ort

$$
\text { Juli } 23,0 \text {. Gr. } x \not \&=221^{\circ} 27^{\prime} 31^{\mu \prime} 2 \delta \mathscr{\&}=+50^{\circ} 43^{\prime} 32^{\mu \prime} 6 \text {. }
$$

Indem ich ohne eine Hypothese taber die Natur des Kegelschnitts aus diesen beiden Örtern und meiner Beobachtung vom 30sten Aug. die Bahn des Cometen bestimmte, fand ich eine der vorigen nahe kommende Ellipse, dereu Elemente sind:

$$
\begin{aligned}
& T=\text { Juni 11,55081 Greenwich } \\
& \pi=249^{\circ} 7^{\prime} 20^{\prime \prime} 6 \\
& \Omega=27858 \quad 8,7\} \text { Mittl. Äquin. 1861,0 } \\
& i=852852,1 \\
& \log q=9,9150472 \\
& \log e=9,9949560 \\
& \log a=1,8525784 \quad \text { Bew. direct. } \\
& \text { Umlaufszeit }=601^{\mathrm{J}} \mathbf{0} \text {. }
\end{aligned}
$$

A. Auwers.

\section{Verkäufliches Passageninstrument.}

Es $_{\mathrm{s}}$ ist bei mir ein im besten Zustande befindliches transportables Passageninstrument vou Elliot, Brothers, zum Verkauf deponirt. Das Fernrohr hat ein achromatisches Objectiv von 20 Pariser Linien Öfnung und 24 Zoll Brennweite, und zwei astrononische Oculare, von denen eins mit einem Prisma versehen ist. Die Fadenbeleuchtnng geschieht durch die Horizontalachse. Die Zapfen sind von Glockenmetall. Das Instrument bat einen Verticalkreis von 5 Zoll Durchmesser, mit zwei Nonien, die unmittelbar Minuten angeben. An dem Nonienträger ist eine Libelle. Eine zweite Libelle dient zum Nivelliren der Hauptachse. Das Stativ ist von Messing. Beigegeben ist noch eine Lampe zur Fadenbeleuchtung. Das Ganze lässt sich in einen dazu gehörigen Kasten verpacken, der nahezu 30 Zoll lang, 16 Zoll breit und 9 Zoll hoch ist. Preis 120 Preuss. Thaler.

\section{I $\mathrm{n}$ h a l $t$.}

(Zu Nr.1325.) Schroiben des Herrn Secchi, Directors der Sternwarte des Coll. Rom, an den Herausgeber 63. Schreiben des Herrn Goldschmidt an den Herausgeber 69 . -$\chi$ Cygni in der Periode der Sichtbarkeit 1861, von Herrn Prof. Heis 69. Beobachtungen am Reichenbach'schen Meridiankreise der Königsberger Steruwarte, von Herrn Sievers 71. Moon Calminations taken at the Observatory Williamstown, Victoria 73. -

Fernere Beobachtungen des Planeten (71) Niobe, von Herrn Dr. R. Luther 75. Einige über Pseudo-Daphne (56), von Herrn Dr. R. Luther 77. -

Flemeite des Cometen II. 1861, von Herrn Auwers 77 -

Verkäufliches Passageninstrument 79. -

Altona 1861. September 11. 